\title{
O método ArcGenx para programação de ônibus urbano e interação com a tabela de horários
}

\author{
Gustavo Peixoto Silva'; Nicolau D. Fares Gualda ${ }^{2}$
}

\begin{abstract}
Resumo: Neste trabalho é apresentada uma versão estendida do método ArcGen, denominada ArcGenX, a qual corresponde a uma incorporação de arcos de auto-atribuição à rede representativa do problema de programação de veículos. O método ArcGen, na forma originalmente apresentada pelos autores, consiste em representar o Problema de Programação de Veículos (PPV) como um problema de circulação numa rede capacitada e resolvê-lo utilizando o algoritmo Out-of-Kilter associado a um processo de geração de arcos. A extensão proposta permite identificar viagens previstas na tabela de horários, cuja eliminação leva à redução da frota de veículos e dos custos operacionais envolvidos. Também permite realizar análises de sensibilidade advindas da flexibilização dos tempos de parada nos terminais. Exemplos de aplicação a casos reais de empresas brasileiras de ônibus são apresentados, com detalhes sobre as conseqüências para a programação dos veículos e as reduções da frota.
\end{abstract}

\begin{abstract}
This paper presents an extended version of the $\operatorname{ArcGen}$ method, called $\operatorname{ArcGenX}$, that incorporates self-attribution arcs to the network representing the vehicle scheduling problem. The method ArcGen, in its original form presented by the authors, consists of representing the Vehicle Scheduling Problem (VSP) as a circulation problem related to a capacitated network and solving it with an arc generation procedure associated to the Out-of-Kilter Algorithm. The proposed extension allows to identifying the fleet and operational costs reduction that can be achieved by eliminating some specific trips of the original timetable. It also allows for sensitivity analyses related to terminal time flexibility. The proposed method was applied to real Brazilian bus cases. Details on the analyses and on the consequences to the vehicle schedules and to fleet reductions are presented.
\end{abstract}

\section{INTRODUÇÃO}

O problema básico de programação de uma frota de ônibus urbano consiste em determinar o número mínimo de veículos necessário para realizar um dado conjunto de viagens, segundo uma tabela de horários pré-definida, assim como definir a seqüência das viagens executadas pelos veículos. Esse problema, dito Problema de Programação de Veículos (PPV), é trivial quando o número de linhas é reduzido, mas se torna complexo à medida que esse número aumenta e é permitido que os veículos realizem viagens em mais de uma linha durante a operação.

Esse problema pode ser formulado com modelos de fluxo em redes de diferentes maneiras. Para melhor compreensão dos diversos modelos, consultar os trabalhos de Daduna e Paixão (1995), Carraresi e Gallo (1984) e Bodin et al. (1983). Independente da formulação adotada, o gargalo na resolução do problema de fluxo subjacente diz respeito ao grande número de arcos na rede. Para contornar este problema, pode-se empregar a técnica de geração de arcos, que consiste em inicializar o domínio do problema com um conjunto de arcos com grande possibilidade de participar da

\footnotetext{
${ }^{1}$ Gustavo Peixoto Silva, Universidade Federal de Ouro Preto, Instituto de Ciências Exatas e Biológicas, Departamento de Computação, Ouro Preto, MG, Brasil. (e-mail: gustavo@iceb.ufop.br).

${ }^{2}$ Nicolau D. Fares Gualda, Universidade de São Paulo, Escola Politécnica, Departamento de Engenharia de Transportes, São Paulo, SP, Brasil. (e-mail: ngualda@usp.br).
}

Manuscrito recebido em 22/9/2008 e aprovado para publicação em 16/6/2009. Este artigo é parte de TRANSPORTES, volume XVII número 1, junho de 2009. ISSN: 1415-7713. solução. Posteriormente, esse conjunto é incrementado com os arcos que apresentam características que permitem melhorar a solução corrente. Assim, é possível atingir a otimalidade sem ter de processar a rede toda (Freling et al., 2001).

O método $\operatorname{ArcGen} X$ é uma extensão do $A r c G e n$ que consiste, basicamente, em transformar a formulação de pseudo-designação do problema (Paixão e Branco, 1987) em um problema de circulação, e resolvê-lo com o algoritmo Out-of-Kilter (Fulkerson, 1961), combinado com a técnica de geração de arcos (Silva, 2001). Entretanto, na extensão apresentada neste trabalho, são acrescidos determinados arcos à rede de circulação que permitem reduzir a frota com a eliminação de determinadas viagens da tabela de horários. Para manter a qualidade do serviço de transporte, são considerados dados como a demanda de passageiros em cada viagem, assim como o intervalo de tempo entre viagens. Desta forma, o modelo integra a programação dos veículos com a tabela de horários, detectando as viagens que, uma vez eliminadas, levam à redução da frota com o mínimo de prejuízo para os usuários do sistema.

As seções a seguir buscam explicitar o método e suas aplicações a um problema real. Assim, na seção 2 é apresentado o PPV como um problema de circulação com custo mínimo. Na seção 3 é feita uma breve descrição do método ArcGen, base para a formulação do método $\operatorname{ArcGenX}$, apresentado na seção 4. Na seção 5 é feita uma descrição do caso estudado, seguida na seção 6, pela aplicação do ArcGen em diferentes cenários. Os resultados obtidos com a extensão $\operatorname{ArcGenX}$ e 
as conclusões do trabalho são apresentados nas seções 7 e 8 , respectivamente.

\section{O PPV COMO PROBLEMA DE CIRCULAÇÃO}

O problema de programação de veículos com uma única garagem (PPV), no qual todas as viagens previstas na tabela horários são executadas impreterivelmente, pode ser definido em termos de fluxo em redes como segue (Gavish et al., 1978; Gavish e Shlifer, 1978).

Dado um conjunto de $n$ viagens, $V=\{1, \ldots, n\}$, cada viagem $i \in V$ é representada por:

- $b_{i}$ o ponto inicial,

- $d_{i}$ o horário de partida de $b_{i}$,

- $e_{i}$ o ponto final e

- $a_{i}$ o horário de chegada em $e_{i}$.

Para qualquer par de viagens $(i, j), t_{i j}$ representa o tempo de viagem em trânsito ou viagem ociosa de $e_{i}$ até $b_{j}$. A garagem é representada pelos nós $r$ e $s$, onde $r$ é o nó de partida da garagem e $s$ é o nó de retorno à garagem, ambos referentes ao mesmo local. Assim, $t_{r i}$ e $t_{i s}$ são os tempos de viagem ociosa (sem carregar passageiros) entre a garagem e os terminais referentes à viagem $i$. Um par de viagens $(i, j)$ é compativel se $d_{j}-a_{i} \geq t_{i j}$, e o custo do arco $(i, j)$ ligando viagens compatíveis $i$ e $j$ é dado por:

$c_{i j}=\left\{\begin{array}{l}K_{1} \cdot t_{i j}+\text { Custo Fixo, se } i=r \\ K_{1} \cdot t_{i j}+K_{2} \cdot(\text { tempo de espera }), \\ \text { se }(i, j) \text { for um par de viagens compatíveis }\end{array}\right.$

sendo $K_{1}$ e $K_{2}$ são constantes associadas aos custos operacionais do veículo, e o tempo de espera é dado por $d_{j}-a_{i}-t_{i j}$. Os valores destas constantes variam de acordo com a situação operacional, entretanto $K_{l}$ é sempre maior do que $K_{2}$ em casos reais. O Custo Fixo é atribuído à primeira viagem de cada veículo, caracterizando a sua utilização e tem como finalidade minimizar o número de veículos na frota.

Este problema normalmente é formulado como um problema de pseudo-designação, no qual as viagens são representadas por dois nós, ou seja, para cada viagem $i \in V$ são definidos os nós $i$ ' de início e $i$ " de final de viagem, com respectiva demanda e oferta de um veículo. A garagem é representada pelo nó $r$ de partida e pelo nó $s$ de retorno, com oferta e demanda de $n$ veículos respectivamente. Os nós são divididos em dois subconjuntos, segundo a oferta ou demanda no nó. Logo, são definidos os subconjuntos: $N_{l}=\{r\}$ $\cup\{i$ ” $\mid i=1, \ldots, n\}$ dos nós com oferta, e $N_{2}=\{s\} \cup$ $\left\{i^{\prime} \mid i=1, \ldots, n\right\}$ dos nós com demanda de fluxo. Os arcos que ligam os nós de $N_{1}$ a $N_{2}$ são definidos por $A=\left\{\left(r, i^{\prime}\right) \mid i=1, \ldots, n\right\} \cup\left\{\left(i^{\prime \prime}, j^{\prime}\right) \mid i, j\right.$ são viagens compatíveis $\} \cup\{(i ”, s) \mid i=1, \ldots, n\} \cup(r, s)$. Assim, tem-se uma rede bipartida $G^{p d}=\left(N_{1}, N_{2}, A\right)$. Neste caso, os custos nos arcos, inicialmente apresentados em (1), ficam da seguinte maneira:

$$
c_{i j}=\left\{\begin{array}{l}
K_{1} \cdot t_{i j}+\text { Custo Fixo, } \\
\text { se } i=r, j=\text { nó início de viagem } \\
K_{1} \cdot t_{i j}+K_{2} \cdot(\text { tempo de espera }), \\
\text { se } i=\text { nó fim de viagem, } j=\text { nó início de viagem }
\end{array}\right.
$$

Para aplicar o algoritmo Out-of-Kilter, a rede que representa o problema deve ser fortemente conectada; desta forma, o modelo de pseudo-designação é transformado em um problema de circulação. Para tanto, são incluídos, na rede, arcos que ligam o nó início ao nó final de cada viagem $i$, com custo nulo e capacidades inferior e superior iguais a um, o que obriga a passagem de uma unidade de fluxo pelo arco $\left(i, i^{\prime}\right)$. Esta imposição garante o atendimento da viagem por um único veículo. Invertendo-se o sentido do arco que liga os nós garagem, ou seja, considerando o $\operatorname{arco}(s, r)$, obtém-se uma representação para o problema por meio de um problema de circulação em uma rede fortemente conectada $G^{c}=\left(N_{l} \cup N_{2}, A\right)$, cujos nós não consomem nem produzem unidades de fluxo. Considerando a rede bipartida $G^{p d}=\left(N_{1}, N_{2}, A\right)$ definida anteriormente, o problema, que pode ser resolvido com o algoritmo Out-of-Kilter, tem a seguinte formulação matemática:

$$
\begin{gathered}
\text { Mín } \sum_{(i, j) \in A} c_{i j} \cdot x_{i j} \text {, sujeito a } \\
\sum_{i \in N_{1} \cup N_{2}}\left(x_{i j}-x_{j i}\right)=0 \quad \forall j \in N_{1} \cup N_{2} \\
x_{i^{\prime \prime} j^{\prime}} \in\{0,1\} \quad \forall\left(i^{\prime \prime}, j^{\prime}\right) \in A-(s, r)
\end{gathered}
$$

em que a variável de decisão $x_{i{ }^{\prime \prime} j^{\prime}}=1$ se a viagem $j$ for executada pelo mesmo veículo imediatamente após a execução da viagem $i$, e $x_{i}{ }^{\prime \prime}{ }^{\prime}=0$ caso contrário. A expressão (3) minimiza o custo total, a restrição (4) garante o equilíbrio de fluxo nos nós, enquanto (5) se refere aos possíveis valores assumidos pela variável de decisão.

Tabela 1. Conjunto de 4 viagens com locais e horários de partida e chegada

\begin{tabular}{ccccc}
\hline Viagem $\boldsymbol{i}$ & $\boldsymbol{d}_{\boldsymbol{i}}$ & $\boldsymbol{b}_{\boldsymbol{i}}$ & $\boldsymbol{a}_{\boldsymbol{i}}$ & $\boldsymbol{e}_{\boldsymbol{i}}$ \\
\hline 1 & $07: 00$ & Term A & $08: 30$ & Term A \\
\hline 2 & $08: 00$ & Term A & $09: 00$ & Term A \\
\hline 3 & $09: 00$ & Term A & $11: 00$ & Term A \\
\hline 4 & $09: 30$ & Term A & $10: 00$ & Term A \\
\hline
\end{tabular}




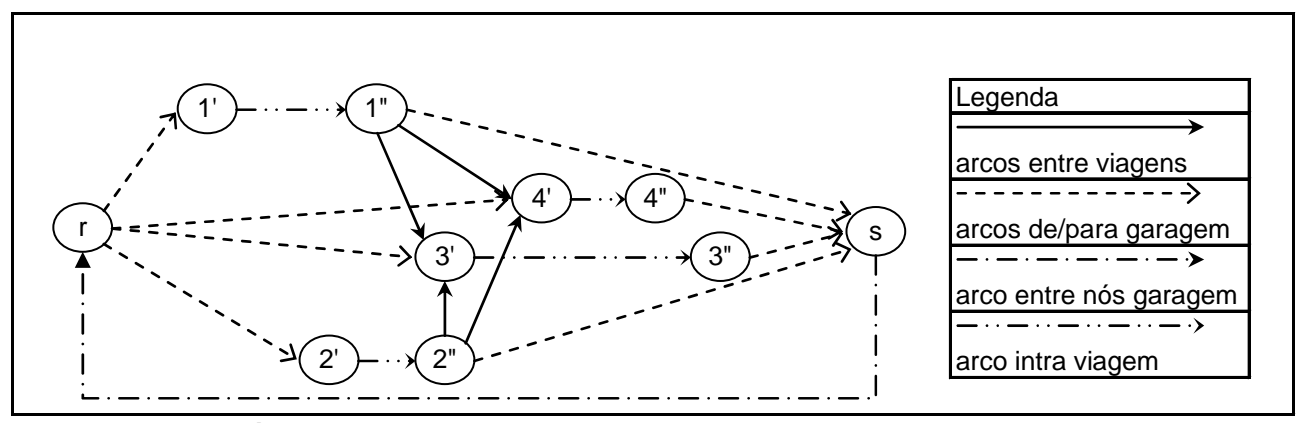

Figura 1. Representação do PPV como um problema de circulação

Para exemplificar o modelo de circulação, considere as viagens contidas na Tabela 1 a seguir.

Para facilitar o entendimento, todas as viagens são iniciadas e finalizadas no mesmo ponto. Após executar a viagem 1 , o mesmo veículo pode ainda realizar a 3 ou a 4, por isso os $\operatorname{arcos}\left(1{ }^{\prime \prime}, 3^{\prime}\right)$ e (1’, 4') fazem parte da rede da Figura 1. O mesmo ocorre com as outras viagens.

A partir da solução deste modelo é possível construir os blocos de viagens para os veículos. Nesta rede, cada caminho que sai de $r$ e chega em $s$ corresponde a um bloco de viagens a ser executado por um dado veículo. O conjunto de todos os caminhos disjuntos de $r$ para $s$, que compõem a solução do problema de circulação dado por (3) a (5), fornece a programação completa com o menor custo fixo e variável possível.

\section{O MÉTODO ArcGen}

Os modelos de fluxo em redes que representam o PPV sempre terão um número elevado de arcos, mesmo para problemas de pequeno porte, comprometendo assim o tempo de processamento computacional para obtenção da solução ótima (Silva et al., 1998). Para contornar este problema, foi desenvolvida uma metodologia que é uma adaptação da técnica de geração de colunas à teoria de grafos (Freling et al., 2001) e a combina com o algoritmo Out-of-Kilter para fluxo em redes (Fulkerson, 1961). Tal implementação deu origem ao método computacional ArcGen para programação de veículos. O método $A r c G e n$ foi aplicado a uma série de casos da realidade brasileira e britânica, mostrando possibilidades de redução da frota de ônibus urbano (Silva et al., 1999; Silva, 2001).

Com uma rede composta por um pequeno conjunto de arcos com grande possibilidade de serem utilizados na solução. Posteriormente é feita uma busca na lista de arcos fora da rede, identificando aqueles com capacidade de melhorar a solução. Se for encontrado algum arco com esta característica, ele é incorporado à rede e o problema de otimização é resolvido novamente. Este processo é repetido até que nenhum arco que está fora da rede possa melhorar a solução. Quando isto ocorrer, o problema está resolvido e a solução corrente é a ótima.
Para aplicar a técnica descrita acima, os arcos da rede são classificados em dois grupos. Os arcos longos ligam duas viagens cujo tempo de espera entre elas é grande o suficiente para que o veículo retorne temporariamente à garagem. Por outro lado, os arcos curtos ligam viagens cujo tempo entre elas não justifica um retorno temporário do veículo à garagem. Assim, define-se o conjunto de arcos longos $A^{l} \subset A$ como sendo os arcos que ligam viagens que satisfazem à seguinte relação:

$$
\begin{aligned}
& (i, j) \in A^{l} \Leftrightarrow \\
& d_{j}-a_{i} \geq t_{i s}+(\text { tempo. mínimo de garagem })+t_{r j}
\end{aligned}
$$

onde o tempo mínimo de garagem é um parâmetro definido pelo operador. O conjunto dos arcos curtos $A^{c}$ é dado por $A^{c}=A-A^{l}$. Embora a maioria dos arcos na rede seja constituída de arcos longos, apenas uma pequena parcela deles participa da solução ótima. Esta característica do problema sugere uma inicialização para a técnica de geração de arcos, descrita a seguir.

O retorno temporário de um veículo à garagem, durante a operação, só se justifica caso o mesmo permaneça no local por um determinado tempo mínimo na garagem definido pelo usuário e que pode ser utilizado para abastecimento e limpeza do veículo. O custo associado ao período em que o veículo permanece temporariamente na garagem é considerado constante e dado por:

$$
\begin{aligned}
& (i, j) \in A^{l} \Rightarrow \\
& c_{i j}=K_{1} \cdot\left(t_{i s}+t_{r j}\right)+K_{2} \cdot(\text { tempo mínimo na garagem })
\end{aligned}
$$

Esta estrutura de custo tem sido largamente adotada (Dell'Amico et al., 1993) e foi incorporada ao modelo ArcGen. A seguir é apresentado, de forma sintética, o método ArcGen para a resolução do PPV.

Inicialização: Faça $S=A^{c}$ e $L=A^{l}$, onde $A$ é o conjunto de todos os arcos da rede que representa o PPV como um problema de circulação.

P1. Resolver o problema de circulação definido em (3)-(5) para a rede $G^{c}=\left(N_{I} \cup N_{2}, S\right)$. Como resultados são obtidos os valores das variáveis primais $x_{i j}$ e duais $\pi_{i}$.

P2. Para cada arco $(i, j) \in L$ :

Calcule $\bar{c}_{i j}=c_{i j}-\pi_{i}+\pi_{j}$ 
Se $\bar{c}_{i j}<0$

Então incluir o $\operatorname{arco}(i, j)$ em $S$ e retirá-lo de $L$.

P3. Se algum arco foi incluído em $S$ durante o passo P2

Então retornar ao passo P1,

Senão finalizar o processo. A solução corrente é ótima.

Desta forma, é possível resolver de maneira exata PPVs de grande porte com uma drástica redução no tempo de processamento.

\section{O MÉTODO ArcGenX: UMA EXTENSÃO DO MÉTODO ArcGen}

O método ArcGen descrito anteriormente encontra a frota mínima necessária para que todas as viagens de uma dada tabela de horários sejam rigorosamente executadas. Por sua vez, o $\operatorname{ArcGenX}$ é um modelo que permite que algumas viagens sejam eliminadas da tabela de horários em função de uma redução na frota e nos custos operacionais. O ArcGenX é uma extensão do ArcGen e sua descrição é feita a seguir.

Considerando o mesmo conjunto de viagens apresentado na Tabela 1 , pode-se construir uma rede adicionando à rede original definida na seção 2 um arco de auto-atribuição para cada uma das viagens da tabela, o qual permite gerar solução com a supressão de viagens programadas. Na Figura 2 é apresentada a rede expandida contendo os arcos de auto-atribuição referentes ao problema exemplo da Tabela 1.

Dada a rede expandida da Figura 2, pode-se aplicar o modelo de circulação com custo mínimo apresentado pelas equações (3) a (5), o qual é resolvido pelo mesmo procedimento adotado no método ArcGen. Resta apenas definir a estrutura de custo para os novos arcos da rede, ou seja, os custos associados aos arcos de auto-atribuição. Considerando esta nova rede, se um arco de auto-atribuição fizer parte da solução, a viagem $i$ referente a ele não será realizada por qualquer veículo da frota mínima. Neste caso, o arco ( $i$, $i$ ”) de realização da viagem $i$ e o seu arco de autoatribuição ( $i$ ”, $i$ ) formam um ciclo isolado, o que significa que um veículo, após realizar a viagem $i$, "retorna no tempo para iniciá-la”. Em outras palavras, pode-se dizer que a viagem $i$ é atendida pelo veículo que acabou de realizá-la, o que não faz sentido na prá- tica. Por outro lado, o arco de realização da viagem $i$, $(i$, $i$ ”) não fará parte de qualquer caminho que liga o nó $r$ de saída da garagem ao nó $s$ de retorno à garagem. Sendo assim, todas as equações de equilíbrio de fluxo da rede são plenamente satisfeitas.

A omissão ou eliminação de algumas viagens da tabela de horários, em função da redução da frota, está associada à relação custo/benefício do sistema como um todo. Neste modelo, viagens com maiores custos nos seus arcos de auto-atribuição são consideradas mais importantes do que viagens com menores custos de omissão, uma vez que a escolha deste arco na solução ótima do problema (de minimização) implica a supressão desta viagem durante a operação.

Neste trabalho são propostas três funções de custo para os arcos de auto-atribuição, que são: $i$ ) custo igual e constante para todas as viagens da tabela de horários, $i i)$ custo dependente do número de passageiros transportados, e iii) custo dependente do número de passageiros e do intervalo até a próxima viagem prevista na tabela. Desta forma, o modelo permite uma integração entre a programação dos veículos e a tabela de horários levando em conta parâmetros de qualidade do serviço prestado.

\section{O CASO ESTUDADO}

O sistema de transporte "Seletivo” de Santos/SP é um serviço diferenciado por apresentar as seguintes características: a) os veículos são de tamanho reduzido (28 assentos) e equipados com ar condicionado; b) os passageiros só viajam sentados; c) o motorista também desempenha a função de cobrador; d) os veículos cumprem suas tabelas de horários com rigidez; e e) o preço da passagem é diferenciado em relação ao transporte coletivo comum. Na época do estudo, o serviço era composto de oito linhas circulares, partindo de três terminais distribuídos na cidade de Santos. A operação do serviço apresenta as seguintes características:

- Para cada linha é definido o número de veículos que nela atuam, tendo em vista a demanda na linha e o tamanho da frota disponível.

- Cada veículo só opera em uma determinada linha.

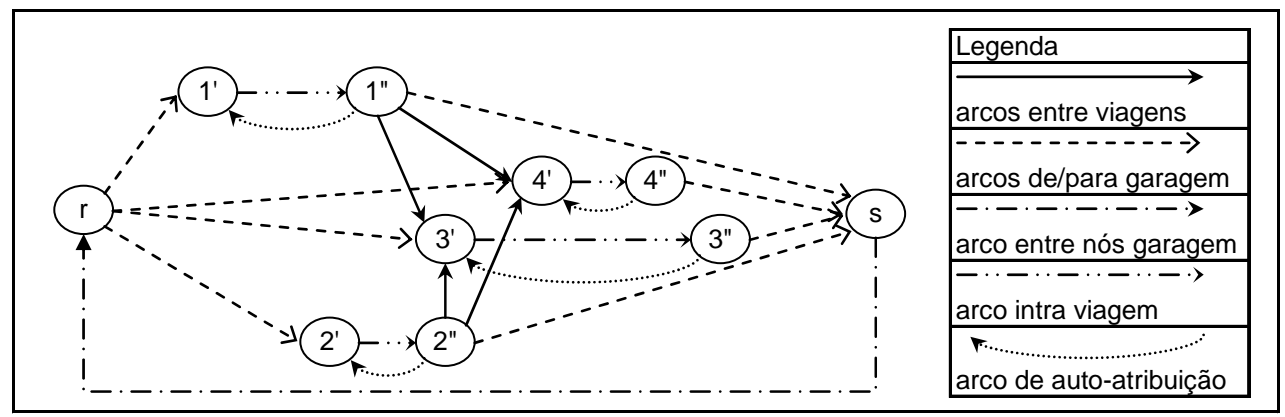

Figura 2. Rede que permite omitir viagens planejadas em função de redução da frota 
- A freqüência de partida de cada linha é calculada dividindo-se o tempo de duração da viagem pelo número de veículos que atuam na linha.

- Ao final de cada viagem, o veículo permanece 10 minutos parado no terminal.

Analisando as características operacionais do serviço de transporte, pode-se concluir que:

- Não é considerada a variação da demanda nas linhas ao longo do dia, visto que a freqüência de partida dos veículos é constante ao longo do dia.

- Ocorre uma super-utilização dos veículos no período de pico e uma sub-utilização no período de entre-pico.

- Não existe retorno de veículos à garagem no período de entre-pico.

- Este serviço segue um padrão operacional de linhas de baixa demanda.

Este trabalho explora as diversas possibilidades de flexibilização no sistema, gerando soluções que permitem uma redução na frota, mas que também requerem mudanças de regras operacionais da empresa. As programações apresentadas representam os principais resultados alcançados e estão de acordo com os parâmetros desejados pela empresa.

Foram realizados dois estudos diferentes, com a finalidade de minimizar a frota em operação. No primeiro caso as viagens da tabela de horário são cumpridas rigorosamente, respeitando os horários iniciais e finais, informados como dados de entrada. Os resultados desta etapa são apresentados na seção 6 e foram obtidos aplicando o método ArcGen na sua forma original. Foram considerados os seguintes cenários: com restrição de troca de linha durante a operação e com liberdade de troca de linha, por parte do veículo, durante a operação. Em ambas as situações, varou-se o tempo mínimo de terminal após cada viagem.

No segundo caso, apresentado na seção 7, o ArcGenX permitiu uma relaxação na execução de todas as viagens contidas na tabela de horários, gerando soluções que omitem algumas viagens em função de uma redução da frota. Neste caso, foram mantidos os mesmos padrões operacionais da empresa, variando a função de custo associada aos arcos de auto-atribuição.

\section{GERAÇÃO DA PROGRAMAÇÃO DE ÔNIBUS E ANÁLISE DA SOLUÇÃO}

Nas programações dos veículos geradas nesta etapa, todas as viagens da tabela de horários são executadas impreterivelmente, respeitando os seus respectivos horários e locais de partida e de chegada. Os dados básicos que alimentaram o sistema foram: o total de via- gens programadas, os horários e locais de início, os horários e locais de término de cada uma das viagens, o tempo de viagem entre os terminais, e o tempo de viagem entre os terminais e a garagem, estando o veículo fora de operação. Também foi informado o tempo mínimo de permanência na garagem, caso o veículo retorne a ela no período de entre-pico, estabelecido em 30 minutos, conforme Kwan e Rahin (1999), e com os quais concordam os gerentes da empresa.

\subsection{Com restrição de troca de linhas}

Nestes casos os veículos são cativos às linhas, realizam sempre viagens de uma mesma linha durante a operação. Quanto ao tempo que o veículo permanece no terminal, após cada viagem, foram testadas duas situações distintas, conforme mostrado nos subitens a seguir.

\subsubsection{Com 10 minutos de tempo no terminal ao final de cada viagem}

Considerando um período mínimo de 10 minutos de terminal após realizar cada viagem, foi gerada uma programação para os veículos com as características apresentadas na Tabela 2.

Tabela 2. Programação sem troca de linha e com tempo de terminal maior que zero

\begin{tabular}{cccc}
\hline Frota & $\begin{array}{c}\text { Tempo total de } \\
\text { viagens vazias }\end{array}$ & $\begin{array}{c}\text { Tempo extra nos } \\
\text { terminais (além dos } \\
10 \text { min previsto }\end{array}$ & $\begin{array}{c}\text { Retorno à } \\
\text { garagem }\end{array}$ \\
\hline 34 & $31: 50 \mathrm{hh}: \mathrm{mm}$ & $00: 00 \mathrm{hh}: \mathrm{mm}$ & 0 \\
\hline
\end{tabular}

Esta programação tem as mesmas características da situação adotada pela empresa e foi gerada para verificar a validade do modelo. Foi verificado que a totalidade das viagens não apresenta folga no terminal além do tempo previsto de 10 minutos no terminal ao final de cada viagem.

\subsubsection{Sem garantia do tempo no terminal ao final de cada viagem}

Se não for imposto ao modelo que os veículos devam permanecer pelo menos 10 minutos no terminal ao final de cada viagem, a programação ótima gerada pelo modelo apresenta as características da Tabela 3, com redução de 2 veículos na frota original.

Tabela 3. Programação sem troca de linha ou garantia do tempo de terminal

\begin{tabular}{cccc}
\hline Frota & $\begin{array}{l}\text { Tempo total de } \\
\text { viagens vazias }\end{array}$ & $\begin{array}{c}\text { Tempo livre nos } \\
\text { terminais }\end{array}$ & $\begin{array}{c}\text { Retorno à } \\
\text { garagem }\end{array}$ \\
\hline 32 & $30: 50 \mathrm{hh}: \mathrm{mm}$ & $44: 50 \mathrm{hh}: \mathrm{mm}$ & 0 \\
\hline
\end{tabular}

Embora esta programação não garanta o tempo de terminal, ela conta com 44 horas e 50 minutos livres nos terminais, o que garante condições operacionais 
da solução. Segundo o histograma de folga, 200 viagens não apresentam folga no terminal. No restante das viagens, ou seja, em 205 viagens, o tempo livre no terminal é de 10 minutos.

\subsection{Com liberdade de troca de linhas durante a operação}

Esta é uma situação de maior flexibilidade, onde um veículo pode realizar viagens de diferentes linhas em um mesmo dia de trabalho. No entanto, o sistema teve seus parâmetros ajustados para que não houvesse um número excessivo de trocas de linhas, mantendo ao máximo as características da programação adotada pela empresa. Assim, como no caso anterior, foram testadas duas situações diferentes para o tempo que o veículo permanece parado no terminal após a execução de cada viagem.

\subsubsection{Com 10 minutos de tempo no terminal ao final de cada viagem}

Impondo ao modelo a restrição de que cada veículo deve permanecer pelo menos 10 minutos no terminal ao final de cada viagem, a programação gerada é apresentada na Tabela 4.

Tabela 4. Programação com troca de linha e com tempo de descanso para o motorista

\begin{tabular}{cccc}
\hline Frota & $\begin{array}{c}\text { Tempo total de } \\
\text { viagens vazias }\end{array}$ & $\begin{array}{c}\text { Tempo extra nos } \\
\text { terminais (além dos } \\
\text { 10 min previstos) }\end{array}$ & $\begin{array}{c}\text { Retorno à } \\
\text { garagem }\end{array}$ \\
\hline 34 & $31: 50$ hh:mm & $00: 00$ hh:mm & 0 \\
\hline $\begin{array}{l}\text { Obs.: Nesta programação os ônibus atuam em média em 3 linhas } \\
\text { diferentes }\end{array}$
\end{tabular}

Neste caso, embora seja garantido o tempo mínimo no terminal, não existe redução no número de ônibus, ocorrendo ainda troca de linhas dos veículos durante a operação.

\subsubsection{Sem garantia de tempo no terminal ao final de cada viagem}

A programação apresentada abaixo não assegura o tempo mínimo de terminal e suas principais características são mostradas na Tabela 5.

Tabela 5. Programação com troca de linha e sem tempo de descanso para o motorista

\begin{tabular}{cccc}
\hline Frota & $\begin{array}{c}\text { Tempo total de } \\
\text { viagens vazias }\end{array}$ & $\begin{array}{c}\text { Tempo livre } \\
\text { nos } \\
\text { terminais }\end{array}$ & $\begin{array}{c}\text { Retorno à } \\
\text { garagem }\end{array}$ \\
\hline 32 & $32: 40$ hh:mm & $32: 20$ hh:mm & $\begin{array}{c}1 \text { (com 02:55 } \\
\text { estacionado }\end{array}$ \\
\hline
\end{tabular}

Obs.: Nesta programação os ônibus atuam em média em 4 linhas diferentes

Embora os veículos operem em diferentes linhas ao longo do horizonte de programação, é verificada uma redução de 2 veículos em uma frota original de 34 veículos, o que torna a solução de interesse prático.

\subsection{Análise dos resultados}

As situações que garantem o tempo mínimo de terminal coincidem com a programação adotada pela empresa, independentemente de haver flexibilização quanto à permanência dos veículos cativos às linhas. Isto se deve ao fato de o intervalo entre as partidas ter sido calculado dividindo-se o tempo de viagem pelo número desejado de veículos em operação nas respectivas linhas.

Nos casos em que não foi imposto ao modelo a permanência do veículo por um tempo mínimo no terminal, houve uma diminuição de 5,88\% na frota alocada. Em contrapartida, surgem as trocas de linhas realizadas pelos veículos durante a operação, o que requer mudança na forma de operação da empresa.

Conforme mencionado anteriormente, o modelo pode ser expandido de tal forma que algumas viagens sejam suprimidas para que haja uma redução no número de veículos. As regras de escolha das viagens a serem suprimidas dependem da função de custo associada. Neste trabalho são propostas três expressões de custo associadas à eliminação de viagens, conforme descrito a seguir.

\section{O PPV INTEGRADO COM A TABELA DE HORÁRIOS VIA ArcGenX}

Nesta seção são apresentados os resultados de uma segunda alternativa para o planejamento das linhas da empresa estudada. Para tanto, o modelo foi expandido para que algumas viagens fossem eliminadas da operação, caso esta eliminação levasse a uma economia significativa, ou seja, a uma redução da frota em operação no sistema. A seguir são detalhadas as extensões necessárias para a eliminação de viagens programadas na tabela de horários.

Nos testes realizados, todas as programações contemplam o tempo de terminal de 10 minutos após o cumprimento de cada viagem, conforme a operação vigente na empresa estudada. Foi considerado também que os veículos não são cativos às linhas, ou seja, podem realizar viagens em diferentes linhas durante a operação.

\subsection{Eliminação de viagens considerando custo constante}

Inicialmente foi considerado um custo de omissão constante e igual para todas as viagens. Desta forma, foram suprimidas viagens que levam à economia de veículos, independentemente das suas características de demanda de passageiros ou de suas inter-relações com as outras viagens da mesma linha. Assim, o custo de omissão de uma viagem é dado por:

$$
C_{o i}=K \text {, para cada viagem } i
$$

onde $K$ é uma constante manipulada pelo usuário para 
controlar o número de viagens suprimidas na programação. Desta forma, caso $K$ assuma valor muito elevado, digamos igual a infinito, então nenhuma viagem será eliminada. Por outro lado, se $K$ for igual a zero, todas as viagens serão suprimidas da tabela de horários. Vale lembrar que o custo $C_{o i}$ corresponde ao custo dado ao arco de auto-atribuição ( $\left.i^{\prime \prime}, i^{\prime}\right)$, arco este que, se fizer parte da solução ótima, representará a supressão da viagem $i$ durante a operação.

No caso analisado, ao assumir um custo de omissão constante para todas as viagens, a retirada de 16 viagens da programação reduz em dois o número de veículos em operação, sendo um veículo da linha 206 e outro da linha 207 (Tabela 6). Algumas viagens destas linhas são incorporadas a dois veículos da linha 202. Por outro lado, as viagens suprimidas estão distribuídas ao longo do dia, abrangendo períodos de pico e fora do pico. O mesmo resultado foi gerado tendo em vista o custo de omissão dado em função da taxa de ocupação dos veículos ( $1^{\circ}$ da tabela 7$)$, porém nesse caso surgem outras programações de interesse prático.

Tabela 6. Programação com custo constante por omissão de viagem

\begin{tabular}{ccc}
\hline Total de veículos & Viagens omitidas & $\begin{array}{c}\text { Total de retorno à } \\
\text { garagem }\end{array}$ \\
\hline \multirow{3}{*}{32 ônibus } & $\begin{array}{c}\text { 8 viagens da linha 206 } \\
\text { (redução de 1 veículo) }\end{array}$ & 0 \\
\cline { 2 - 3 } & $\begin{array}{c}\text { 8 viagens da linha 207 } \\
\text { (redução de 1 veículo) }\end{array}$ & \\
\hline $\begin{array}{c}\text { Obs.: Dois ônibus operam em duas linha diferentes, realizando quatro } \\
\text { viagens em uma segunda linha }\end{array}$
\end{tabular}

\subsection{Eliminação de viagens considerando a taxa de ocupação do veículo}

Para serem considerados fatores da demanda de passageiros, foram associadas ao custo de omissão informações referentes à taxa de ocupação do veículo em cada uma de suas viagens. Assim, o período de operação de cada linha foi dividido em intervalos caracterizados pela demanda, tais como: pré-pico, pico da manhã, pós-pico e entre-pico. A empresa forneceu, para cada intervalo, a taxa de ocupação do veículo. Esta ta- xa, que reflete a demanda na linha, foi incorporada ao custo de omissão da viagem. Foi utilizado, ainda, um multiplicador para controlar os custos e regular o número de viagens omitidas, como pode ser verificado na expressão a seguir.

$$
C_{o i}=K \cdot D_{i} \text {, para cada viagem } i
$$

onde $K$ é a constante a ser controlada pelo usuário e $D_{i}$ representa a taxa média de ocupação do veículo para a viagem $i$.

Considerando-se a demanda nos custos de omissão das viagens, as soluções mais interessantes são aquelas nas quais os veículos realizam alguma troca de linha durante a operação. As duas primeiras programações na Tabela 7 utilizam 32 veículos, excluindo 16 e 17 viagens respectivamente. Na primeira programação, apenas dois veículos operam em duas linhas diferentes, realizando um bloco de 4 viagens ao final do período. No caso da segunda programação, quatro veículos atuam em duas linhas diferentes, sempre em blocos de 3 a 4 viagens, no início ou no final do período. Esta programação suprime 17 viagens e, portanto, uma a mais do que a programação anterior. A vantagem é que as viagens não realizadas estão distribuídas em períodos fora do horário de pico das respectivas linhas.

As duas últimas programações da Tabela 7 utilizam 33 veículos, omitindo, em cada caso, 8 viagens. Na terceira programação, todas as viagens não realizadas pertencem à linha 206, abrangem horários de pico e fora do pico, e um único veículo da frota atua em linhas diferentes. Esse veículo realiza um bloco de 4 viagens na linha secundária ao final do período de operação. Na programação de número 4, as viagens não realizadas pertencem a duas linhas diferentes, 206 e 207, não se encontram em horários de pico, e dois veículos atuam em linhas diferentes, realizando blocos de viagens na linha secundária no início ou no final do período de operação.

Tabela 7. Programações com custo por omissão em função da taxa de ocupação dos veículos

\begin{tabular}{|c|c|c|c|c|}
\hline \multirow{2}{*}{$\begin{array}{l}\text { Número veículos } \\
\text { Total de omissões }\end{array}$} & \multirow{2}{*}{$\begin{array}{c}\text { Linha/Viagens omitidas } \\
- \\
\text { período das viagens }\end{array}$} & \multicolumn{3}{|c|}{ Veículos com troca de linha } \\
\hline & & Linha principal & $\begin{array}{c}\text { Linha secundária } \\
x \\
n^{o} . \text { de viagens } \\
\end{array}$ & $\begin{array}{l}\text { Período viagens } \\
\text { secundárias }\end{array}$ \\
\hline $\begin{array}{c}32 \text { / } 16 \\
\text { Programação } 1\end{array}$ & $\begin{array}{l}\text { 206/8 viagens - entre } 07: 30 \text { e } 17: 25 \text {. } \\
\text { 207/8 viagens - entre } 07: 50 \text { e } 17: 45\end{array}$ & $\begin{array}{l}202 \\
202\end{array}$ & $\begin{array}{l}206 \times 4 \\
207 \times 4\end{array}$ & $\begin{array}{l}\text { fim do período } \\
\text { fim do período }\end{array}$ \\
\hline $\begin{array}{c}32 \text { / } 17 \\
\text { Programação } 2\end{array}$ & $\begin{array}{l}\text { 208/3 viagens - entre 07:05 e 09:45. } \\
\text { 207/4 viagens - entre 07:50 e 12:05. } \\
\text { 206/10 viagens - entre 11:05 e 18:10. }\end{array}$ & $\begin{array}{l}201 \\
202 \\
207 \\
208 \\
\end{array}$ & $\begin{array}{l}206 \times 3 \\
206 \times 4 \\
206 \times 4 \\
206 \times 3\end{array}$ & $\begin{array}{l}\text { fim do período } \\
\text { fim do período } \\
\text { início do período } \\
\text { início do período }\end{array}$ \\
\hline $\begin{array}{c}33 / 8 \\
\text { Programação } 3 \\
\end{array}$ & 206/8 viagens - entre 07:30 e 17:25 & 202 & $206 \times 4$ & fim do período \\
\hline $\begin{array}{c}33 \text { / } 8 \\
\text { Programação } 4\end{array}$ & $\begin{array}{l}\text { 207/4 viagens - entre 7:50 e 12:05. } \\
\text { 206/4 viagens - entre 13:10 e 17:25. }\end{array}$ & $\begin{array}{l}202 \\
207\end{array}$ & $\begin{array}{l}206 \times 4 \\
206 \times 4\end{array}$ & $\begin{array}{l}\text { fim do período } \\
\text { início do período }\end{array}$ \\
\hline
\end{tabular}




\subsection{Eliminação considerando a taxa de ocupação do veículo e intervalo entre viagens}

Outro fator que reflete a qualidade de um serviço de transporte público é o intervalo entre as partidas dos veículos de uma dada linha, denominado headway da linha. Se, por um lado, o intervalo mais amplo entre duas partidas aumenta a taxa de ocupação do veículo, por outro lado ele caracteriza uma escassez de opções para o usuário. Este fator foi incorporado ao modelo atribuindo-se ao custo de omissão de cada viagem a expressão abaixo, que consiste no produto entre a taxa de ocupação do veículo e o intervalo de tempo até a próxima partida, além do fator multiplicativo.

$$
C_{o i}=K \cdot D_{i} \cdot T_{i} \text {, para cada viagem } i
$$

onde $K$ e $D_{i}$ têm a mesma interpretação da expressão (9) e $T_{i}$ é o intervalo de tempo até a próxima partida da linha a que pertence a viagem $i$.

A Tabela 8 mostra as programações resultantes, tendo em vista os custos expressos em (10). Foram apresentadas apenas aquelas que mais se aproximam da programação corrente, e em todas as soluções ocorre troca de linha por parte dos veículos.

Para reduzir a frota ao mesmo número de veículos (32 e 33) das programações apresentadas na Tabela 7, as programações desta seção suprimiram um número maior de viagens. No primeiro caso, para uma frota de 32 ônibus, foi necessário suprimir um total de 19 viagens, portanto 3 e 2 viagens a mais do que nas duas primeiras programações da Tabela 7. Por outro lado, no máximo 3 veículos fazem troca de linhas, correspondendo à média em relação aos dois primeiros casos da tabela anterior. A segunda programação da Tabela 8 apresenta resultado semelhante aos das programações 3 e 4 da Tabela 7, porém neste caso foi necessário eliminar 9 viagens e, portanto, uma a mais. Entretanto, a solução apresenta um espaçamento mais uniforme na tabela de horários resultante.

\subsection{Análise dos resultados}

A Tabela 7 apresenta dois grupos de programações com a mesma taxa de redução da frota, porém com diferentes características operacionais. Enquanto na primeira e na terceira programações algumas das via- gens omitidas pertencem ao horário de pico das respectivas linhas, as viagens eliminadas na segunda e na quarta programações pertencem ao horário de entrepico. Em contrapartida, existe um número menor de veículos operando em linhas diferentes na primeira e na terceira programações do que na segunda e na quarta programações.

Embora os resultados apresentados na Tabela 8 suprimam algumas viagens a mais do que nos casos anteriores, para as mesmas dimensões da frota (32 e 33 veículos), adotando-se a função de custo de omissão de viagens na expressão (11) foi possível gerar outras alternativas de programação. A primeira e a segunda programações da Tabela 8 podem ser comparadas à segunda e à quarta programações da Tabela 7 , pois ambos os pares têm a mesma frota e todas as viagens omitidas estão fora do horário de pico. A diferença qualitativa entre estes resultados, além do número de viagens omitidas, está nas linhas que sofrem redução de viagem, no número de veículos que atuam em linhas diferentes e no número viagens realizadas na linha secundária.

Comparando as programações com 32 veículos pode-se observar que: a) existe uma interseção entre elas de apenas três viagens da linha 208; b) 4 e 3 veículos atuam em linhas diferentes nas programações da Tabela 7 e Tabela 8, respectivamente; e c) são realizadas 14 e 12 viagens nas linhas secundárias nas programações da Tabela 7 e Tabela 8, respectivamente.

Para as programações com 33 veículos observa-se que: a) as programações omitem viagens pertencentes a linhas distintas; e b) são realizadas 8 e 6 viagens nas linhas secundárias nas programações da Tabela 7 e Tabela 8, respectivamente. Logo, são gerados resultados alternativos e de certa forma complementares para uma mesma dimensão da frota, propiciando flexibilidade de escolha ao operador do sistema de transporte.

Cabe ressaltar que os tempos de computacionais para a resolução dos problemas apresentados neste trabalho são da ordem de 2 segundos, utilizando um computador pessoal com processador Pentium 4.0, 2.8 GHz e 2.0 GB de memória RAM.

\section{CONCLUSÕES}

Neste trabalho foi apresentado inicialmente o método

Tabela 8. Programações que consideram a taxa de ocupação e o intervalo entre partidas

\begin{tabular}{|c|c|c|c|c|}
\hline \multirow{2}{*}{$\begin{array}{c}\text { Número veículos } \\
\text { Total de omissões }\end{array}$} & \multirow{2}{*}{$\begin{array}{c}\text { Linha/Viagens omitidas } \\
- \\
\text { período das viagens }\end{array}$} & \multicolumn{3}{|c|}{ Veículos com troca de linha } \\
\hline & & Linha principal & $\begin{array}{c}\text { Linha secundária } \\
x \\
n^{o} . \text { de viagens } \\
\end{array}$ & $\begin{array}{l}\text { Período viagens } \\
\text { secundárias }\end{array}$ \\
\hline \multirow{3}{*}{$\begin{array}{c}32 \text { / } 19 \\
\text { Programação } 1\end{array}$} & \multirow{3}{*}{$\begin{array}{l}\text { 208/9 viagens - entre 07:05 e 12:25. } \\
\text { 204/10 viagens - entre 13:05 e 18:10. }\end{array}$} & 208 & $204 \times 6$ & Início do período \\
\hline & & 202 & $208 \times 1$ & Início do período \\
\hline & & 208 & $204 \times 5$ & Início do período \\
\hline $33 / 9$ & 208/4 viagens - entre 08:00 e 12:00. & 202 & $208 \times 1$ & Início do período \\
\hline Programação 2 & 204/5 viagens - entre 13:05 e 17:45. & 208 & $204 \times 5$ & Início do período \\
\hline
\end{tabular}


ArcGen e uma aplicação que resolve o problema clássico de programação de veículos, o PPV. O método minimiza a frota necessária e também os custos operacionais envolvidos na execução de um conjunto de viagens contidos na tabela de horários. As soluções obtidas para o problema analisado levaram à redução de 2 veículos de um total de 34 utilizados pela empresa. Esta redução pode ser obtida considerando os veículos cativos às linhas ou permitindo que estes operem em mais de uma linha durante suas jornadas.

O método ArcGen foi estendido, gerando um modelo ainda inédito na literatura, denominado pelos autores de $\operatorname{ArcGen} X$, que integra o problema de programação dos veículos com a tabela de horários. Esta integração possibilita suprimir algumas das viagens previstas na tabela de horários, permitindo gerar programações que reduzem ainda mais a frota empenhada na operação. Os resultados mais interessantes do ponto de vista operacional são aqueles que determinam as viagens a serem eliminadas da tabela de horários respeitando a taxa de ocupação dos veículos e aqueles que levam em conta a taxa de ocupação do veículo e o intervalo até a próxima viagem na decisão de eliminar viagens da tabela de horários. Desta forma, são omitidas viagens pouco produtivas e que não comprometem a qualidade do serviço prestado.

Assim, com a aplicação do método ArcGen, no qual todas as viagens previstas na tabela de horário são necessariamente realizadas, a redução da frota pode ser alcançada com a mudança na forma de operação dos veículos, reduzindo-se o tempo de terminal dos mesmos e/ou permitindo que os veículos atuem em mais de uma linha no mesmo dia. Já a aplicação do método $\operatorname{ArcGenX}$ permite manter os mesmos tempos de terminal praticados pela empresa e ainda reduzir a frota com a eliminação de algumas viagens. A eliminação dessas viagens pode ser controlada pelo usuário levando em conta fatores de produtividade dos veículos e qualidade do serviço oferecido.

\section{AGRADECIMENTOS}

Os autores agradecem ao CNPq e à FAPEMIG pelos apoios recebidos.

\section{REFERÊNCIAS BIBLIOGRÁFICAS}

Bodin, L.; B. Golden; A. Assad e M. Ball. (1983) Routing and scheduling of vehicles and crews: The state of the art. Computers and Operations Research, v 10, p. 63-211.

Carraresi, P. e G. Gallo (1984) Network models for vehicle and crew scheduling. European Journal of Operational Research, v 16, p. 139-151.

Daduna, J. R. e J. M. P. Paixão (1995) Vehicle scheduling for public mass transport- an overview. In: Daduna, J. R.; I. Branco e J. M. P. Paixão (eds.) Computer-Aided Transit Scheduling, Lectures Notes in Economics and Mathemaitcal Systems. Springer Verlag, Berlin, Alemanha.

Dell'Amico, M.; Fischetti, M. e P. Toth (1993) Heuristic algorithm for the multiple depot vehicle scheduling problem. Management Science, v.39, p.115-125.
Freling, R.; J. M. P. Paixão e A. P. M. Wagelmans (2001) Models and Algorithms for Vehicle Scheduling. Transportation Science v. 35, p 165-180.

Fulkerson, D. (1961) An out-of-kilter method for minimal cost flow problems. SIAM Journal on Applied Mathematics, v.9, p.18-27.

Gavish, B.; P. Schweitzer e E. Shlifer (1978) Assigning buses to schedules in a metropolitan area. Computers and Operations Research, v.5, p. $129-138$

Gavish, B. e E. Shlifer (1978) An approach for solving a class of transportation scheduling problems. Computer and Operations Research, v. 3, p. 122-134.

Kwan, R. K. e M. A. Rahin (1999) Object oriented bus scheduling - the BOOST system. Computer Aided Scheduling of Public Transport (ed.) N. H. M. Wilson, p. 179-191. Springer, Berlin.

Paixão, J. e L. Branco (1987) A quasi-assignment algorithm for busscheduling. Networks, v 17, p. 249-269.

Silva, G. P. (2001) Uma metodologia baseada na técnica de geração de arcos para o problema de programação de veículos. Tese de doutorado. Escola Politécnica da USP, São Paulo.

Silva, G. P.; R. S. K. Kwan e N. D. F. Gualda (1998) Vehicle scheduling with network flow models. Transportes, v. 6, p. 6-27.

Silva, G. P.; A. Wren; R. S. K. Kwan e N. D. F. Gualda (1999) An arc generation approach to solving the bus scheduling problem. Research Report Series, Report 99.01. School of Computer Studies, University of Leeds, Reino Unido. 